\title{
HISTORIA DE LA LITOTRICIA POR ONDAS DE CHOQUE EN ESPAÑA
}

Francisco Javier Ruíz Marcellán y Luis Ibarz Servio.

Centro de Litiasis Renal. Servicio de Urología. Instituto Universitario Dexeus. Barcelona. España.

Resumen.- Se aporta bosquejo histórico acerca de la urolitiasis con especial hincapié en las alternativas históricas a la cirugía de la litiasis.

Se introducen los antecedentes que llevan al nacimiento de la litotricia extracorpórea por ondas de choque y a la implantación de la misma en nuestro país.

Palabras clave: Litiasis renal. Litotricia. Ondas de choque. Historia de la Urología.
Summary.- We give a historical outline of urinary lithiasis with emphasis in the alternative therapeutic options to surgery.

We expose the previous steps that led to the birth of extracorporeal shockwave lithotripsy and its implementation in our country.

Keywords: Renal lithiasis. Lithotripsy. Shock waves. History of Urology.

\section{UROLOGIA}

La Urología como especialidad médico-quirúrgica se afianzó en las primeras décadas del siglo XX a consecuencia de la aparición de determinadas técnicas de exploración y tratamiento que requerían un especial aprendizaje y experiencia.

El diagnóstico urológico se basó durante muchos años en las exploraciones endoscópicas, el cateterismo de los uréteres con separación de orinas y la pielografía ascendente.

En 1929, el Servicio de Urología de Alexander von Lichtenberg en Berlín, publicaba los resultados iniciales de la experiencia clínica sobre la urografía de excreción, el método daba una información de gran interés cuando la cistoscopia y el cateterismo ureteral eran imposibles.

Gran parte de la cirugía urológica reparadora ha sido posible gracias al conocimiento de la enorme capacidad de recuperación renal tras un proceso obstructivo, y éste más perfecto conocimiento se deriva de la experiencia obtenida con la urografía intravenosa. 
La cirugía endoscópica fue otro gran avance de la Urología. McCarthy (1932), Nesbit (1939) e Iglesias (1948) desarrollaron los resectoscopios con sus vainas, ópticas de fibra de vidrio, corriente de libre circulación y pedal de doble mando de coagulación y corte. La aplicación de la cirugía endoscópica consolidó en los alrededores de 1950 la especialidad de Urología en Estados Unidos y Europa.

La evolución paulatina de la cirugía urológica reparadora infantil y del adulto, el desarrollo del trasplante de órganos, la arteriografía con el catéter de Seldinger, los isótopos radioactivos (renograma y gammagrafía renal), han supuesto la maduración y el florecimiento de la especialidad.

\section{UROLOGÍA Y LITIASIS URINARIA}

La historia de la urolitiasis es aparentemente tan vieja como la humanidad. En la tríada de operaciones electivas inicialmente practicadas por el hombre -circuncisión, trepanación y talla vesical-, sólo la última no iba unida a un ritual religioso, lo que la convierte en la cirugía más antigua dirigida a la curación de un mal específico.

El objeto de interés urológico más antiguo que se recuerda es un cálculo vesical de aproximadamente 7000 años (4800 años a de C), descubierto por E. Smith en 1901 en una tumba prehistórica de El Amrah, cerca de Abydos.

Hipócrates hizo una precisa descripción de la litiasis con la semiología del cólico nefrítico y sus teorías sobre la litogénesis.

Celso, que vivió en el primer siglo de nuestra era, describió exhaustivamente la técnica de la litotomía perineal. Incindía la vejiga sin referencias anatómicas, atravesando todo el espesor del periné hasta la piedra.

En 1520, una nueva técnica de litotomía fue introducida por Marianus Sanctus. Primero pasaba por la uretra una herramienta o itinerarium para contactar con la piedra, incindía la uretra membranosa e introducía dentro de la vejiga el exploratorium, el cual guiaba un fórceps que atraía la piedra hacia el exterior.

A finales del siglo XVIII la talla lateralizada era el único método de litotomía en uso tanto en Francia como en Inglaterra. Jean Civiale comienza sus experimentos en 1817 cuando todavía era un estu- diante de segundo año en la Universidad de París, en orden a obtener la destrucción de los cálculos en la vejiga sin dañar sus paredes, para luego eliminar el paciente los fragmentos del cálculo con la micción. Sobre él recae el honor de haber llevado a cabo la primera litotricia transuretral el 13 de Enero de 1824 en el Hospital Necker de París.

Los resultados del método francés fueron excelentes y aprobados por Bigelow en la Universidad de Harvard que abogó por la fragmentación y extracción completa de los restros calculosos introduciendo el término de litolapaxia.

En el siglo XX con el desarrollo de la anestesia, la antisepsia, la endoscopia y los grandes avances en el conocimiento aplicado al estudio y tratamiento de la litiasis, se consolida el desarrollo médico, tecnológico y quirúrgico del modo de tratar la enfermedad litiásica.

Henri Morris, en 1880, realizó la primera nefrectomía por litiasis y definió con precisión los términos de nefrolitiasis, nefrolitotomía, nefrectomía y nefrotomía.

Fundamental fue la aportación de José $M^{a} \mathrm{Gil}$ Vernet en 1960, que desarrolló la vía intrasinusal de acceso a la pelvis renal y cálices, evitando lesionar el parénquima renal y su vascularización. Significativas son también las aportaciones de Smith y Boyce en la cirugía conservadora de la litiasis coraliforme.

Paralelamente a los avances quirúrgicos toman especial atención en este siglo los estudios de la fisiopatología de la litiasis urinaria. El papel relevante de los factores locales renales en la nucleación de los cálculos fue extensamente estudiado por Randal, que descubrió en 1937 las placas que llevan su nombre.

La hipercalciuria como factor contribuyente a la formación de los cálculos fue definida por Flocks en 1939. La hipercalciuria del hiperparatiroidismo fue identificada por Flocks en 1940 y Albright en 1948

Cifuentes y De Vries, de forma separada, sistematizaron el tratamiento alcalinizante de los cálculos de ácido úrico.

El creciente interés por el estudio de los factores médicos y la prevención de la litiasis propició la creación de unidades y laboratorios especializados, siendo el pionero en nuestro país el de los doctores Cifuentes y Rapado en la Fundación Jiménez Díaz de Madrid. 


\section{ALTERNATIVAS A LA CIRUGÍA DE LA LITIASIS. CONSIDERACIONES HISTÓRICAS.}

El intento de evitar la cirugía abierta de la litiasis se ha materializado en tres líneas de actuación:

a. Disolución de los cálculos in situ o litolisis química

b. Endourología transuretral o translumbar para la aplicación de diferentes formas de energía: electrohidraúlica, ultrasónica, láser y neumática. Todas ellas incluídas en la acepción de litotricia intracorpórea o de contacto.

c. Litotricia extracorpórea por ondas de choque.

a. Litolisis: La disolución local mediante irrigación de diferentes sustancias líticas ha sido un intento muy meritorio durante varias décadas del siglo XX. Suby, Mulvaney, Timmermann, Dormia, Thomas, Pérez Castro, etc han sido los autores que más destacaron en este campo.

Los cateterismos prolongados con graves infecciones, las irritaciones químicas de la mucosa y otras graves complicaciones desestimaron este método. La quimiolisis local ha quedado limitada a algunos restos mucoproteícos postcirugía o litotricia de coraliformes complejos. Por vía general mantiene vigencia el tratamiento lítico de la litiasis úrica y cistínica.

b. Endourología: Ureteroscopia y nefrolitotomía percutánea. La ureteroscopia transuretral fue introducida por E. Pérez-Castro en 1980 permitiendo el dominio bajo visión directa de la totalidad del uréter hasta la pelvis renal y la fragmentación y extracción de los cálculos ureterales entre otros procedimientos.

Alken y Wickham en 1981, trabajando separadamente en Alemania e Inglaterra, desarrollaron y establecieron la metodología de la cirugía percutánea renal como alternativa a la cirugía convencional de la litiasis.

En los últimos 25 años se han desarrollado diferentes energías de fragmentación por contacto como son los ultrasonidos, la electrohidraúlica, el láser y la energía neumática, utilizadas tanto en litotricia intracorpórea vesical, ureteral o renal.

c. Litotricia extracorpórea por ondas de choque. El conocimiento de las ondas de choque de alta energía proviene de antiguo. Por ejemplo, los efectos de las ondas expansivas de las explosiones sobre los diferentes órganos de los seres vivos.
La técnica de la litotricia extracorpórea por ondas de choque es consecuencia de la investigación aplicada con fines militares. Durante la Segunda Guerra Mundial, algunos bombarderos B-29 se desintegraron en el aire. Las averiguaciones realizadas por ingenieros aeronáuticos norteamericanos revelaron que los accidentes se produjeron durante temporales. Experimentos de simulación en el laboratorio demostraron que los impactos de las gotas de lluvia sobre el fuselaje del avión se comportaban como micrometeoritos que provocaban ondas de alta energía, las ondas de choque, con suficiente energía como para pulverizar materiales frágiles. El problema se resolvió modificando el temple del cristal de la cabina del piloto.

Al terminar la guerra, esta información quedó archivada y olvidada hasta que, a finales de la década de los 50, los aviones a reacción empezaron a tener los mismos problemas. En un centro de pruebas de Alemania Occidental, ingenieros de la compañía aeronaútica Dornier lanzaron gotas de agua a una velocidad ocho veces superior a la del sonido contra ciertos elementos de un caza Lockheed F-104. "Las gotas hicieron unos cráteres de 20 milímetros de profundidad en los extremos de las alas y otras superficies del aparato. Y las ondas de choque generadas por estos impactos rompieron la burbuja de la cabina", comentó Günter Hoff, físico que participó en el proyecto. En el transcurso de esas investigaciones, en el año 1966, se descubrió la transmisión inocua de las ondas de choque mecánicas a través del cuerpo. Un ingeniero tocó una cartulina de uso diario en el mismo momento en que recibía un impacto de un proyectil de alta velocidad, sintió una especie de shock eléctrico pero sin evidencia de fenómenos eléctricos reales.

Tras recomendar varios cambios en el diseño de tales burbujas, los investigadores de Dornier, a requerimiento de médicos interesados, se prestaron a estudiar las posibles aplicaciones de las ondas de choque en Medicina.. Pero, primero, tenían que inventar una forma de generar y controlar la energía para que ésta pudiese ser dirigida hacia una zona determinada del cuerpo.

De esta forma, a partir de 1969, se desarrollaron técnicas para reproducir las ondas de choque en el laboratorio. Así, diseñaron un generador de ondas de choque cuyo fundamento se basa en la producción de una chispa eléctrica en una cápsula elipsoidal sumergida en el agua. Cuando salta la chispa entre los dos polos del electrodo crea una onda de choque que la cápsula refleja hacia un punto distante. De este modo, la energía puede transmitirse a través de agua o de los tejidos vivos hasta un 
blanco frágil, en este caso un cálculo renal, sin daño demostrable, excepto en el caso del pulmón.

Después de esta primera etapa era preciso idear un sistema de localización de la litiasis y un sistema mecánico que permitiera situar el cálculo en el punto focal del hemielipsoide reflector. Las primeras tentativas de localización fueron hechas con ultrasonidos pero no lograron obtener una precisión suficiente por lo que se eligió un sistema radiológico convencional. La ubicación espacial del cálculo en las tres dimensiones se obtuvo con la utilización de un sistema biaxial de Rayos $X$.

La fase de experimentación animal se desarrolló en Munich entre 1971 y 1978 . En este largo período de experimentación "in vitro" e "in vivo" sobre cálculos, tejidos y animales de experimentación, se comprobó la fragmentación de los cálculos y se descartó la aparición de lesiones graves en los tejidos vecinos. Para el desarrollo de un prototipo experimental de litotritor con posibilidades de aplicación en humanos fue necesaria la aprobación del proyecto por el Ministerio de Investigación y Tecnología de la entonces República Federal Alemana, lo que ocurrió en 1974.

El desarrollo de la investigación se debió en un principio a los profesores Eisenberger y Chaussy, y continuada por este último en el Instituto de Investigación Quirúrgica de la Universidad de Munich.

Después de siete años de investigación y desarrollo se realizó en 1980 por Chaussy en el Hospital Universitario de Munich la primera litotricia extracorpórea en humanos. En los dos años siguientes fueron tratados 200 pacientes con cálculos renales, que

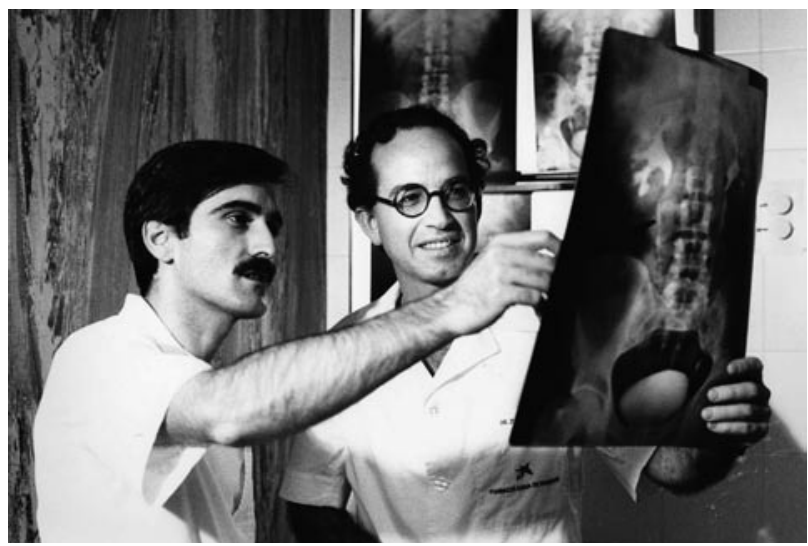

FIGURA 1. Los Dres. Ruíz Marcellán e Ibarz comentando un caso el 8-7 7-84, inicio de la LEOC en el país. eran destruidos por acción de las ondas de choque y sus fragmentos resultantes eliminados por la orina de forma espontánea. Las pruebas de función renal no mostraron cambios morfológicos ni funcionales en el riñón tratado ni en los órganos adyacentes. En 1982, se inauguró en dicho hospital la primera Unidad de Litotricia Extracorpórea por Ondas de Choque.

Con la instalación del segundo litotritor Dornier HM-3 en 1983 en el Katarinen Hospital de Stutgart (Alemania) se empieza a reconocer el papel de la litotricia en el tratamiento de la litiasis renal y se comienza a producir este aparato en serie. Después de su aprobación, en 1984, por la FDA americana en los años siguientes el método se introdujo en los principales hospitales del mundo estableciéndose la litotricia como método de elección en el tratamiento de la litiasis renal.

\section{CENTRO SANITARIO DE LITIASIS RENAL}

El 8 de noviembre de 1984 se realizó el primer tratamiento de litotricia extracorpórea por ondas de choque en España en el Centro Sanitario de Litiasis Renal (Instituto Dexeus) de Barcelona. El tratamiento fue practicado con éxito por los Dres F.J. Ruiz Marcellán y L. Ibarz Servio (Figura 1).

El litotritor Dornier HM-3 (Figura 2) había sido adquirido por la Fundación Caja de Pensiones con un coste de 350.000 .000 de pesetas e instalado como unidad autónoma, el Centro Sanitario de Litiasis Renal dirigido por el Dr Ruiz Marcellán, dentro del Instituto Universitario Dexeus de Barcelona. Previamente, el equipo asistencial (médicos urólogos, anestesiólogos y enfermeras) habían completado un

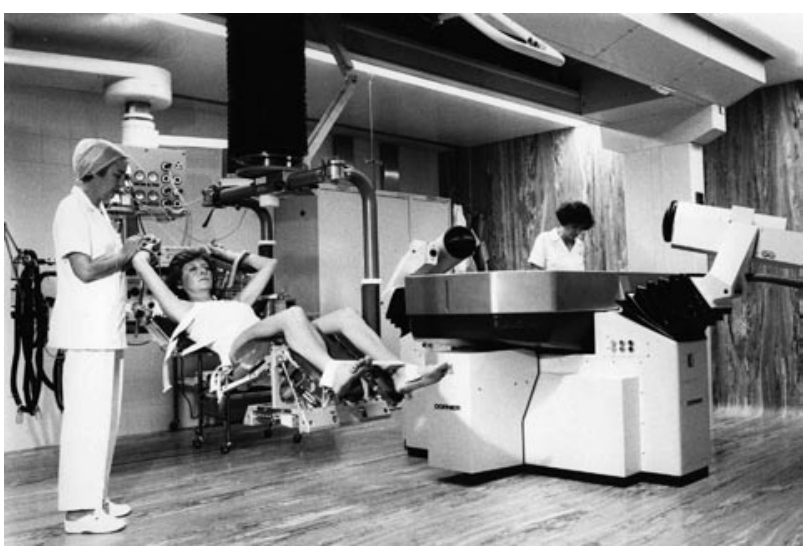

FIGURA 2. Dispositivo de colocación (camilla) y acoplamiento (bañera) del Litotritor Dornier HM-3. 


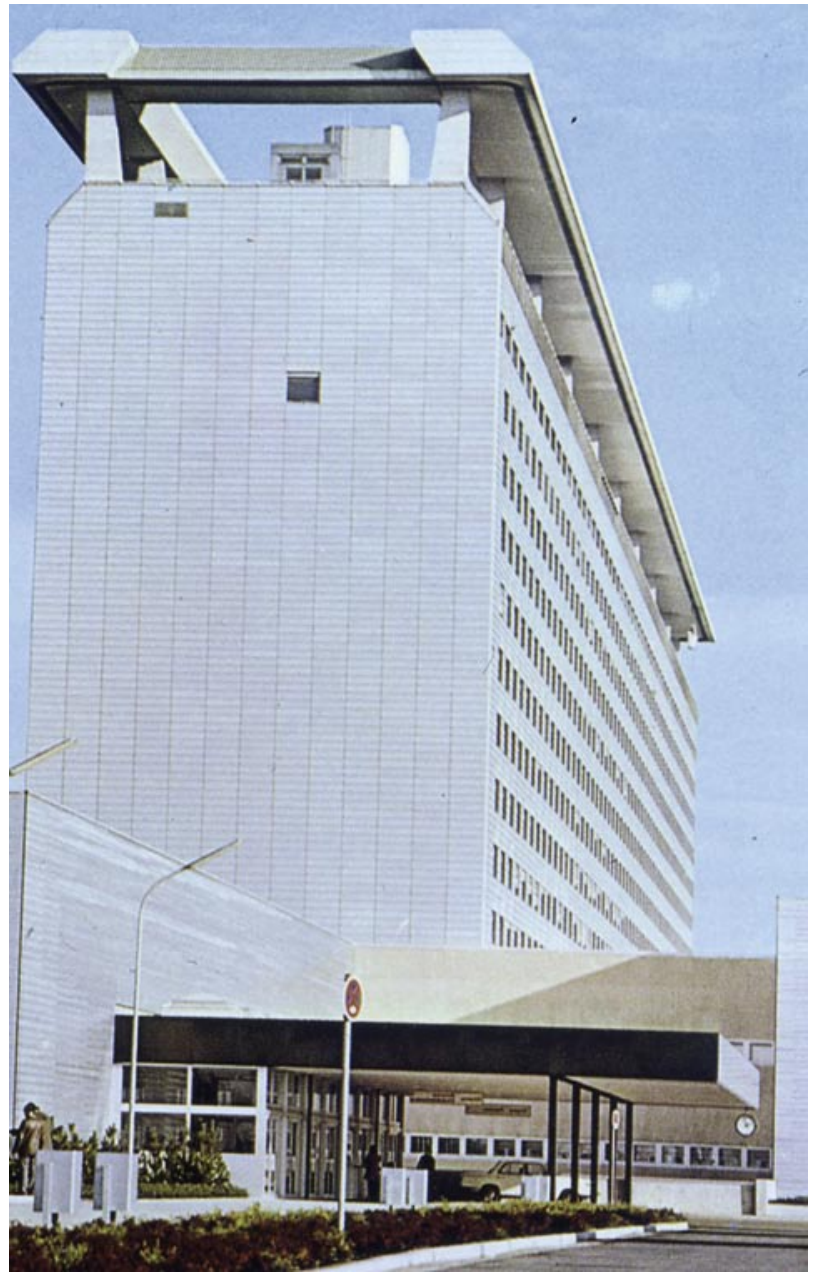

FIGURA 3. Fachada del Hospital Universitario de Munich.

período de formación en el Hospital Universitario de Munich Klinikum Grosshadern (Figura 3) junto al profesor Ch. Chaussy (Figura 4).

El Centro se dio a conocer mediante la organización de las "Jornadas Internacionales de Avances en Urolitiasis" que tuvieron lugar del 13 a 15 de Abril de 1984 (Figura 5). Siempre bajo los auspicios de la Fundación Caja de Pensiones se dieron cita en el Museo de la Ciencia de Barcelona más de 200 urólogos de todo el país para asistir a las conferencias dictadas por algunos de los mejores especialistas en litiasis de toda Europa (Figura 6). En el programa se abordaron tanto los aspectos médicos epidemiológicos, análisis estructural de los cálculos, estudio metabólico mineral, hipercalciurias, litiasis infectiva y tratamiento de las alteraciones metabólicas, como los aspectos instrumentales y quirúrgicos de las ondas de choque, la endourología y la cirugía de las litiasis más complejas.

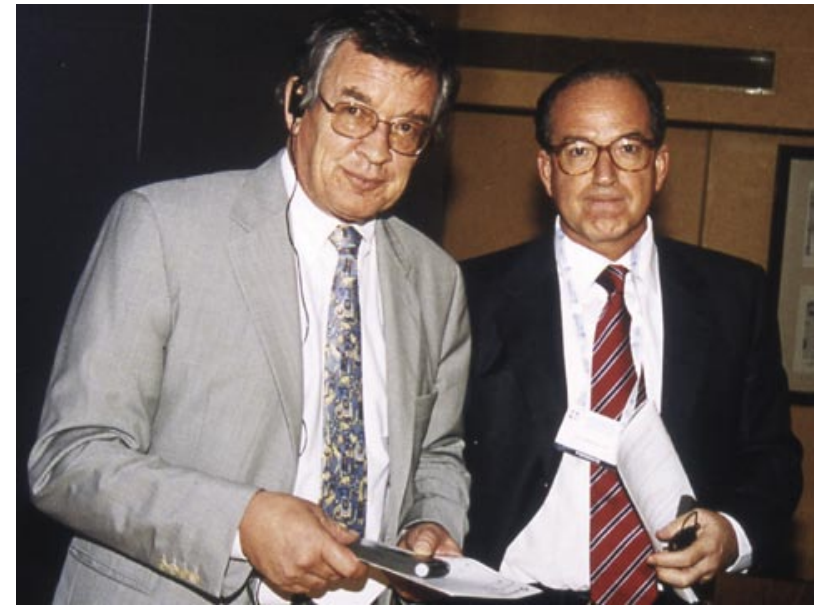

FIGURA 4. Los Dres. Chaussy y Ruíz Marcellán.

La organización de las jornadas tuvo además de la consiguiente repercusión profesional una amplia difusión mediática. En los diarios de aquellos días podían leerse opiniones tan contrapuestas como "la bañera de Munich no resuelve todos los casos y las informaciones superficiales al respecto pueden hacer que muchos pacientes retrasen su intervención" o que "dada su eficacia... estoy convencido de que dentro de unos años habrá un litotritor en todos los grandes centros de la Seguridad Social". El hecho cierto es que desde finales de 1985 a 1987 se introdujeron

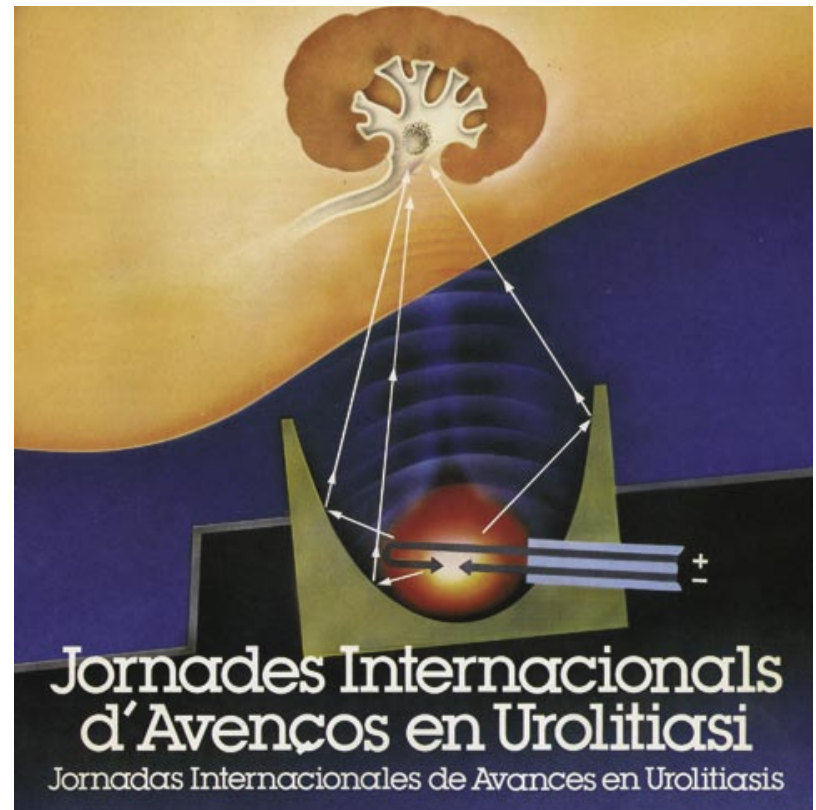

FIGURA 5. Programa de las Jornadas Internacionales de Avances en Urolitiasis, Abril de 1984. 

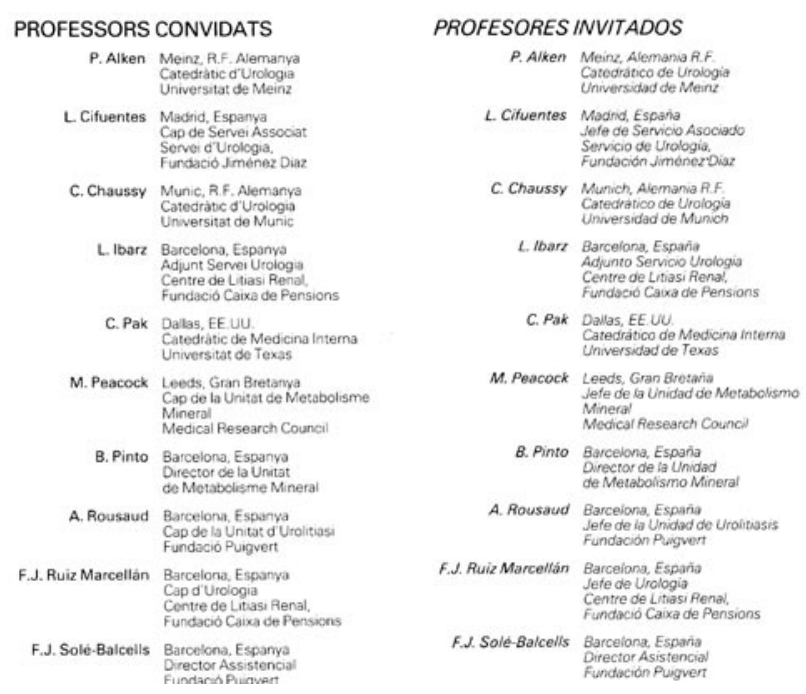

FIGURA 6. Profesores invitados a las Jornadas Internacionales de Avances en Urolitiasis.

varias máquinas de litotricia por toda la geografía española (Sevilla, Madrid, Valencia, Zaragoza, etc) y el método pasó a ser reconocido y financiado por la sanidad pública dada la gran demanda que se generó por parte de los pacientes a favor de la "bañera de Munich" y en contra de la cirugía abierta.

El Centro Sanitario de Litiasis Renal (Figura 7) ha continuado trabajando durante casi 25 años en el

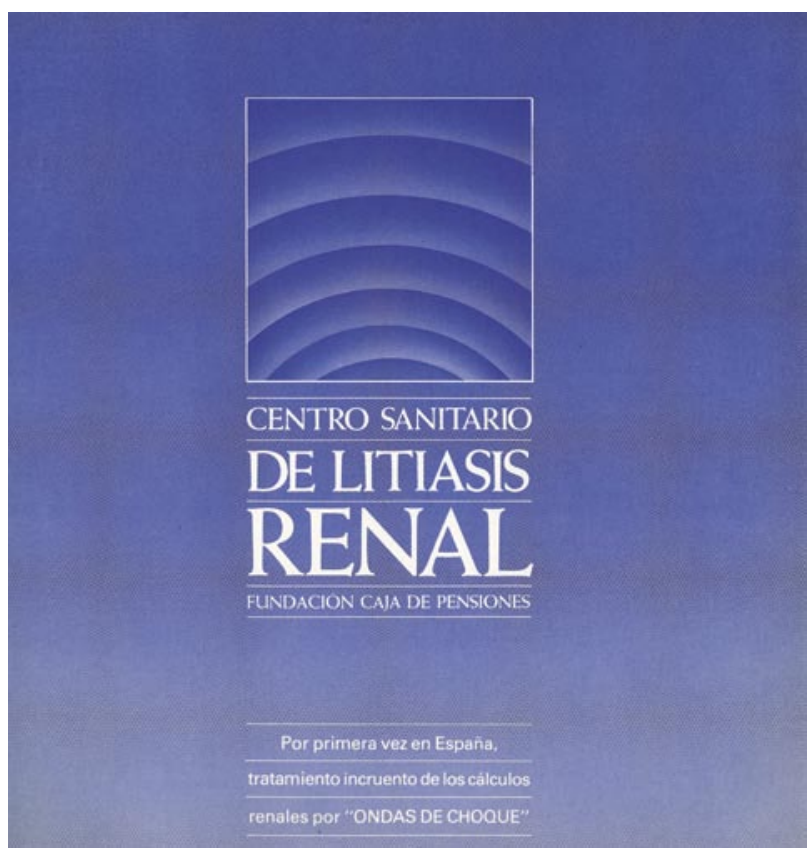

FIGURA 7. Folleto explicativo del Centro Sanitario de Litiasis Renal. estudio y tratamiento integral del enfermo litiásico. Su objetivo ha sido siempre doble: reducir al máximo las intervenciones quirúrgicas mediante la aplicación de la litotricia extracorpórea por ondas de choque y la endourologia, y evitar la recidiva litiásica mediante el estudio y la corrección de las alteraciones metabólicas.

Desde la fundación del Centro se han atendido más de 30.000 pacientes litiásicos que han requerido varios miles de sesiones de litotricia y cientos de ureteroscopias y nefrolitotomías percutáneas. Igual que en otras unidades el litotritor Dornier HM3 original ha sido sustituído por nuevas tecnologías en producción de ondas de choque, básicamente la energía electromagnética que sustituye a la electrohidraúlica, y que, mejorando la ergonomía, la aplicación indolora de las ondas y generalizando los tratamientos ambulatorios, inclusive el costo económico, no siempre se ha traducido en una mayor eficacia desintegradora.

La situación de la litotricia a comienzos de nuestro siglo ha sido ampliamente recogida y documentada por el Grupo de Litiasis de la Asociación Española de Urología siendo coordinador el Dr Arrabal. En el 2000 existían 60 unidades funcionales, 23 del sistema sanitario público. Para una población de casi 40 millones de habitantes esto supone un equipo de litotricia por cada 660.000 habitantes, proporción similar a la de EEUU, inferior a la de Alemania y bastante superior a la de Francia.

Se han tratado unos 300.000 casos observándose una disminución progresiva de los grandes cálculos renales a favor de cálculos más pequeños y sobre todo ureterales. La media de sesiones de litotricia ha sido de 1,7 y se ha precisado tratamiento quirúrgico abierto o endoscópico en el 10,6\% de los pacientes. La valoración del tratamiento ha sido de éxito completo en el $79 \%$ de los casos, parcial en el $13,8 \%$ y de fracaso terapéutico en el 7,2 . Las complicaciones observadas en orden descendente de frecuencia han sido el cólico renal en $28 \%$, obstrucción severa $4 \%$, sepsis $1,1 \%$, hematoma retroperitoneal $0,4 \%$ y pérdida de función renal $0,4 \%$.

Para concluir, creemos que el panorama actual del tratamiento de la litiasis es esperanzador sobre todo cuando se atiende al problema litiásico desde una visión integral que no solo se limita al tratamiento del cálculo sino también a la prevención de la recidiva. 